\title{
An Efficient Method to Restore Torn Digital Image with Enhanced Resolution
}

\author{
Shadiya $\mathbf{N}^{1}$, Ameer $\mathrm{Ali}^{2}$ \\ ${ }^{1,2}$ Department of Computer Science and Engineering, KMCT College of Engineering, Calicut
}

\begin{abstract}
A tear results in local image data loss and a relative shift in the frame between the regions at either side of the tear boundary. This paper proposes algorithms for the digital restoration of images damaged by tear. It describes a method for delineating the tear boundary and for correcting the displacement. For a high-quality image restoration, a missing-data treatment algorithm is used to recover any missing pixel intensities. To construct a high resolution image of the restored image, an interpolation technique is also proposed in this paper. Image interpolation aims in reconstructing a high resolution $(H R)$ image from one or more low resolution (LR) images. It is better to explore the linear relationship among neighboring pixels to reconstruct a high-resolution image from a lowresolution input image. This paper uses an efficient method to determine the local order of the linear model implicitly. According to this theory, a method for performing single image super resolution is proposed by formulating the reconstruction as the recovery of a low rank matrix, which can be solved by the augmented Lagrange multiplier method. In addition, the proposed method can be used to handle noisy data and random perturbations robustly.
\end{abstract}

Keywords: Convex Combination, Image Interpolation, Low rank matrix recovery, Restoration

\section{Introduction}

Over the past two decades, there has been an increasing desire among broadcasters and film studios to commercially exploit their archives. Due to the often poor condition of these archives and the modern expectation for high-quality digital video, a demand exists for automatic archive restoration. This demand has resulted in much research into algorithms for the digital restoration of archived media among the image and video processing community. Most of the research effort has focused on commonly occurring impairments such as film-grain noise, film unsteadiness, film flicker, line scratches and dirt and sparkle.

This paper deals with film tear; the automatic correction of which has not been addressed previously in the community. A tear is caused by the physical ripping of the film material, which divides the film into two. The two main problems resulting from film tear are the relative displacement of the regions at either side of the tear and the destruction of image data along the tear boundary. The destruction of image data requires a missing-data treatment solution that is similar to the restoration of film degraded by dirt and sparkle. However, applying a missing-data treatment algorithm to a torn frame would still result in a visible impairment as relative displacement would cause a spatial discontinuity in the edges that cross the tear boundary.

Previously, tear has been restored by manually joining film sections using a transparent adhesive tape or by using imageediting software to manually realign the two regions. These methods can be painstaking and are prone to failures such as subsequent stretching and damage to the splice. Since manual digital correction tends to ignore the temporal context of the torn frame, it is unable to resolve tear displacement accurately in cases where the edges crossing the boundary have predominantly one orientation. Furthermore, such manual techniques do not address the problem of data loss along the tear boundary. Therefore, there is a demand for digital restoration that could perform the film realignment with greater reliability and less user intervention. Unlike artifacts such as noise and dirt, tear occurs infrequently in archive sequences if at all. Consequently, the computational efficiency demands are not as great for tear restoration as they are for the restoration of other artifacts.

Image super-resolution (SR) technology is always desirable in visual information processing to obtain more detail in an image. It aims to reconstruct a high resolution (HR) image from one or more low-resolution (LR) images. This task essentially can be converted into an inverse problem of the image degradation process. However, the SR problem is inherently ill-posed because many $\mathrm{HR}$ images can generate the same LR image by down-sampling. Therefore, prior knowledge and fundamental assumptions are necessary to obtain high-quality HR images from LR ones. This prior knowledge and these assumptions with respect to image capture, such as the type of motion, blurring, and quality, usually come from common sense or statistical laws. Later, various methods have been proposed to stabilize the SR illposed problem.

In the proposed project, a technique is used to digitally restore a detected torn image. This technique involves tear delineation, displacement correction and recovery of the damaged image data. Then the project involves an image interpolation technique [1] to construct high resolution of the restored image. It also includes a method to handle noisy data and random perturbations robustly.

\section{Related Works}

Research attempt related to Image super resolution (SR) technology have employed a number of techniques and methods focusing mainly on providing high quality HR images from LR ones. Some noticeable works in this area are as follows: 


\section{International Journal of Science and Research (IJSR) \\ ISSN (Online): 2319-7064}

Index Copernicus Value (2013): 6.14 | Impact Factor (2015): 6.391

An example-based method was proposed by W. T. Freeman, T. R. Jones, and E. C. Pasztor [2], which assumes that lost high-frequency details in a low resolution image can be learned from trained low resolution and high resolution patch pairs. That is, the high resolution image can be obtained by learning the co-occurrence relationship between these training patch pairs. However, if the training samples used are unsuitable, example-based SR methods may produce obvious artifacts and unwanted noise in the synthesized image.

In [3], K. Zhang, X. Gao, D. Tao, and X. L. Li, proposed a method in which super resolution is achieved by learning both non-local and local regularization priors from a given low-resolution image. The non-local prior uses the redundancy of similar patches in natural images and the local prior assumes that a target pixel can be estimated by a weighted average of its neighbors. Based on this concept, the non-local means filter is used to learn a non-local prior and the steering kernel regression to learn a local prior. By combining the two regularization terms, a maximum a posteriori probability framework is proposed for SR recovery. This method can reconstruct higher quality results both quantitatively and perceptually. The problem of this method is it doesn't consider/self-similar redundancy both within the same scale and across different scale.

In [4], Yang et al. proposed a sparse representation SR method. This method can choose the most relevant reconstruction neighbors adaptively and thus avoid over- or under-fitting. Here, the low-resolution image is viewed as down-sampled version of a high-resolution image, whose patches are assumed to have a sparse representation with respect to an over-complete dictionary of prototype signal atoms. Under mild conditions, the principle of compressed sensing ensures that the sparse representation can be correctly recovered from the down-sampled signal. A small set of randomly chosen raw patches from training images of similar statistical nature to the input image serve as a good dictionary, in the sense that the computed representation is sparse and the recovered high-resolution image is superior in quality to images produced by other SR methods. However, the problem is to determine, in terms of the within-category variation, the number of raw sample patches required to generate a dictionary satisfying the sparse representation prior.

In practice, it is difficult to obtain more than one low resolution image for the same scene. Example, in the case of recovery of old photos, restoration of calligraphy, handwriting authentication, paintings. So, the single image super-resolution (SISR) problem is more practical and valuable. It is also difficult to make use of the limited information in one low resolution image to reconstruct high resolution image. As the paper focuses on the situation where low resolution image is directly down-sampled from the original high resolution image, the SISR problem turns into an image-interpolation problem The following papers focuses on the image-interpolation problem.

A classical bilinear interpolation method is proposed by $\mathrm{X}$. Zhang and $\mathrm{X}$. Wu, [5] for reconstructing a high resolution image from a low-resolution input image. This method takes a weighted average of the 4 neighborhood pixels to calculate its final interpolated value. The result is much smoother image. If the distances all known pixel are equal, then the interpolated value is simply their sum divided by four. This technique performs interpolation in both directions that is in horizontal and vertical directions. Bilinear interpolation technique gives better result than nearest neighbor interpolation. And it takes less computation time compared to bicubic interpolation.

In [6], H. S. Hou and H. C. Andrews proposed bicubic interpolation method. In bicubic interpolation it considers the closest $4 \times 4$ neighborhood of known pixels for a total of 16 pixels. The distances to known pixels from the unknown pixel are different, so higher weighting is given to the closer pixels in the calculation. In this method, initially four cubic polynomials are fitted to the control points in the y-direction (the choice of starting direction is arbitrary). Then, the fractional part of the calculated pixel's address in the ydirection is used to fit another cubic polynomial in the $\mathrm{x}$ direction, based on the interpolated brightness values that lie on the curves. By substituting the fractional part of the calculated pixel's address in the x-direction into the resulting cubic polynomial then produces the interpolated pixel's brightness value. This method provides noticeably sharper images than the bilinear interpolation methods, and is perhaps the ideal combination of processing time and output quality. Because of this reason it is a standard used in many image editing programs including Adobe Photoshop, printer drivers and in-camera interpolation.

$\mathrm{Fu}$ et al. [7] proposed matrix completion method which is used to solve the SISR problem by efficiently exploring the linear relationship among neighboring pixels is a pervasive way to reconstruct high-resolution image from low resolution one. It is a challenge to determine the order of linear model. According to the theory of matrix completion, it proposes a single frame super-resolution algorithm by minimizing the sum of all the augmented matrices' rank, which can reflect the order of the region aware linear model. Due to the local structural similarity of the images, the augmented matrix has low rank. That is, the center pixels can be represented by the 8 -connected neighboring pixels or a subset of the 8connected neighboring pixels. Experiments demonstrate the images reconstructed by the proposed method have superior PSNR and visual quality, benefitting from its desirable ability of depressing the ringing noise and other artifacts. The Problem of this method is due to the presence of noise and random perturbations, some entries in the augmented matrix are corrupted.

In the above method, all the images are noise-free and perfectly aligned with each other at the pixel level. But, in real world scenarios, small noise and misalignment are very common in any data acquisition process. The matrix completion (MC) problem can be viewed as a case of the matrix recovery problem, in which one has to recover the missing entries of a matrix with given limited number of known entries. In [8], Z. Lin, M. Chen, L. Wu, and Y. Ma, proposed a method of augmented Lagrange multipliers (ALM) to solve the matrix completion problem. The ALM

\section{Volume 5 Issue 5, May 2016}




\section{International Journal of Science and Research (IJSR) \\ ISSN (Online): 2319-7064}

Index Copernicus Value (2013): 6.14 | Impact Factor (2015): 6.391

algorithm is faster than the SVT algorithm. And it is simpler to analyze and easier to implement. Moreover, it provides higher accuracy as the iterations are proven to converge to the exact solution of the problem. Finally, ALM algorithms require less storage/memory.

However, the SISR problem can be represented as that of recovering and completing a low-rank augmented matrix (MCR) in the presence of random perturbations and noise. This problem can be expressed as a rank minimization Problem. The problem can be solved by the augmented Lagrange multiplier method (ALM). The method proposed by Feilong Cao and Miaomiao Cai, [1] overcomes all the problem faced by the traditional interpolation In this method, SISR reconstruction is formulated as a rank minimization problem. HR image can be recovered by solving the rank minimization problem.

\section{Restoration of Torn Image with Enhanced Resolution}

The proposed method restores the image which is damaged by tear, in high quality. The restoration process [9] involves tear delineation, displacement correction and missing data treatment. Tear delineation process is used when detected torn frames are segmented into two regions divided by the tear boundary. The task called displacement correction is used to correct the relative displacement between the segmented regions. Missing data Treatment is used for the recovery of damaged image data.

After the restoration process, an image interpolation technique is applied over the restored image. In interpolation, it is better to explore the linear relationship among neighboring pixels. The technique used here uses an efficient method to determine the local order of the linear model implicitly. According to this theory, a method for performing single image super resolution is proposed by formulating the reconstruction as the recovery of a low rank matrix, which can be solved by the Augmented Lagrange multiplier method.

In addition to the restoration of torn image with high quality, the proposed framework also includes a method to restore an image which is affected by noise. That is, if the input image is a noisy one, then the framework uses a denoising algorithm to remove the noise content from the image. The denoising algorithm used here is Block Matching 3- Dimensional (BM3D) [10], which is based on an enhanced sparse representation in transform domain. The enhancement of the sparsity is achieved by grouping similar 2-D image fragments into 3-D data arrays which is called as groups. Collaborative filtering is a special procedure developed to deal with these 3-D groups. It includes the three successive steps: 3-D transformation of a group, shrinkage of the transform spectrum, and inverse 3-D transformation. After denoising the image, convex combination of original noisy image and de-noised image is performed. Convex combination [11], is a linear framework which is used to obtain the high quality HR image. This linear combination is performed on orientation and frequency selective bands of the two images.
Then the same interpolation technique is applied to get noisefree high resolution image.

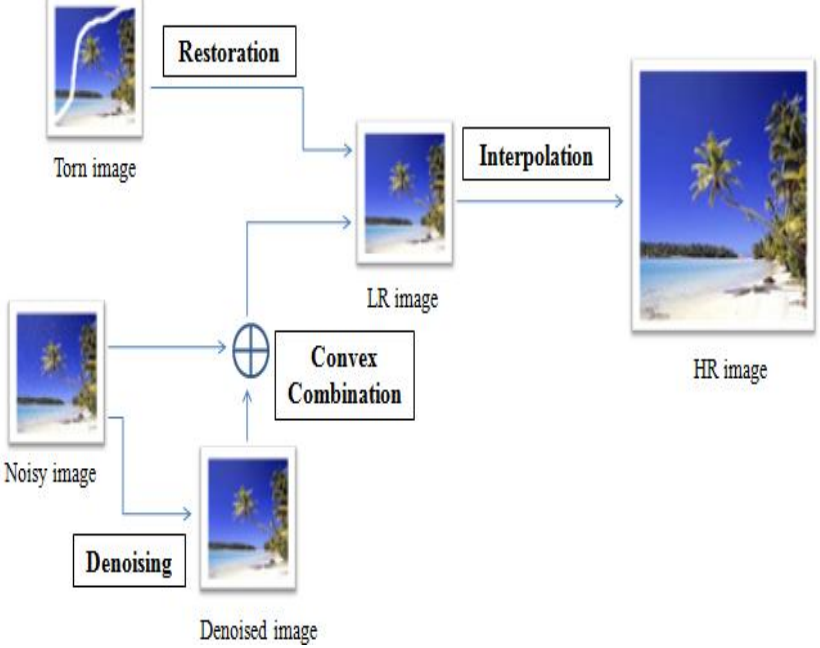

Figure 1: The Framework of Restoration of Torn Image with enhanced Resolution

\subsection{Restoration of Torn Image}

Digital Restoration algorithm is used to restore the images damaged by tear. This Restoration is performed through the execution of sequence of process. Initially in this restoration process, motion vector of each pixel in the torn image is estimated. Motion vector is estimated based on the histogram of the pixel. Then following tasks must be performed:

\subsubsection{Tear Delineation}

It is used when detected torn frames are segmented into two regions divided by the tear boundary. This task involves Motion Histogram segmentation, Tri-Map Refinement and Graph Cut Segmentation [12]. Motion Histogram segmentation is done to decompose the image into subunits for further processing. In this stage, watershed algorithm [13] is used to find dominant modes of motion. To ensure no pixels are assigned to incorrect region, Tri-Map Refinement is performed. Tri-Map Refinement is a two stage erosion process. In the first stage, different values are assigned to the pixel and in second stage; a conventional noise removal algorithm is used. In this phase, hard constraints are identified. Finally, Graph Cut segmentation is used to resolve unassigned region. At the end of this task, the exact tear boundary will be identified.

\subsubsection{Displacement Correction}

It is used to correct the relative displacement between the segmented regions. This task involves Regional Model Estimation and Regional Model Compensation. Regional Motion Estimation is based on the motion vector of the pixel and the outcome of Graph Cut segmentation. In this, Global motion of the image and local motion of the pixel are estimated and compared. For finding Regional Motion Compensation, first order linear equation is applied over affine model of the image. So at the end of this phase, displacement is estimated. 


\section{International Journal of Science and Research (IJSR) \\ ISSN (Online): 2319-7064 \\ Index Copernicus Value (2013): 6.14 | Impact Factor (2015): 6.391}

\subsubsection{Missing Data Treatment}

It is used for the recovery of damaged image data. An integrated Bayesian approach is used here.

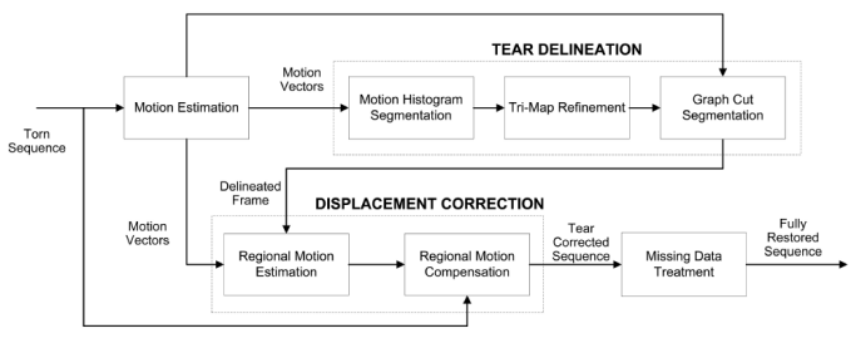

Figure 2: Steps involved in the restoration.

\subsection{Image Interpolation}

After Restoration process, high resolution the restored image is constructed by using image interpolation via Low Rank Matrix Completion and Recovery method [1]. In this method, SISR reconstruction is formulated as a rank minimization problem. The high resolution image can be constructed by solving the rank minimization problem. In the recovery process, the color image is first transformed from RGB color space to $\mathrm{YCbCr}$ color space. For the color channels $\mathrm{Cb}$ and $\mathrm{Cr}$, the bicubic interpolation method is used to upsample them. In the $\mathrm{Y}$ channel, the proposed low rank matrix recovery method is used to estimate the missing pixels of the local window,. The processing of the local windows is in raster scan order in the image. That is, by processing the local windows from left to right and from top to bottom compatibility between adjacent local windows is enforced.

In this method, interpolations of the missing pixels are performed in two phases. There are three kinds of pixels: solid dots, shaded dots, and empty dots. The solid dots are the known LR pixels. The shaded and empty dots are the missing pixels. In the first phase, initial estimates of the empty dots are obtained by using bilinear interpolation method. Then the solid dots and the empty dots are used to recover the shaded dots by using low rank matrix recovery theory. In the second phase, using low rank matrix recovery theory the final values of the empty dots is revised. This method improves the effect of reconstruction both visually and numerically. At the end of this phase, a restored high resolution image is obtained.

\subsection{Denoising of Image}

If the input image is a noisy one, then the framework uses a denoising algorithm to remove the noise content from the image. The denoising algorithm used here is Block Matching 3- Dimensional (BM3D), which is based on an enhanced sparse representation in transform domain. The enhancement of the sparsity is achieved by grouping similar 2-D image fragments into 3-D data arrays which is called as groups. Collaborative filtering is a special procedure developed to deal with these 3-D groups. It includes the three successive steps: 3-D transformation of a group, shrinkage of the transform spectrum, and inverse 3-D transformation. The result is a 3-D estimate that consists of the jointly filtered grouped image blocks. By attenuating the noise, the collaborative filtering reveals even the finest details shared by grouped blocks and, at the same time, it preserves the essential unique features of each individual block. The filtered blocks are then returned to their original positions. Because these blocks are overlapping, for each pixel, many different estimates are obtained which need to be combined. Aggregation is a particular averaging procedure which is exploited to take advantage of this redundancy. A significant improvement is obtained by a specially developed collaborative Wiener filtering. After denoising, convex combination is performed.

\subsection{Convex Combination}

Convex combination is a linear framework which is used to obtain the high quality HR image. The inputs to the module are original noisy input image and the denoised copy of that image. While also containing noise, the noisy image contains some of the textural components which are not present in the de-noised image due the de-noising loss. In order to obtain a noise-free image that also contains these textural details, a linear framework that obtains the desired image as a convex combination of the de-noised image and the noisy HR image is used. This linear combination is performed on orientation and frequency selective bands of the two images. After convex combination, the same interpolation technique is applied to get noise-free high resolution image.

\section{Implementation and Analysis}

The Restoration of Torn image with enhanced Resolution is implemented using Matlab. The testing of the proposed method is done by using images of different dimensions. The proposed method is appropriate for all type of images.

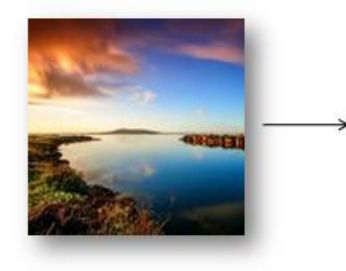

Low Resolution Image

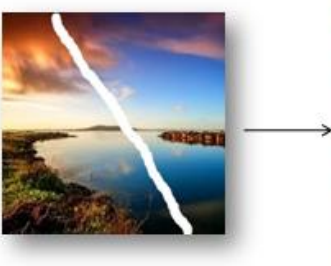

Torn Image

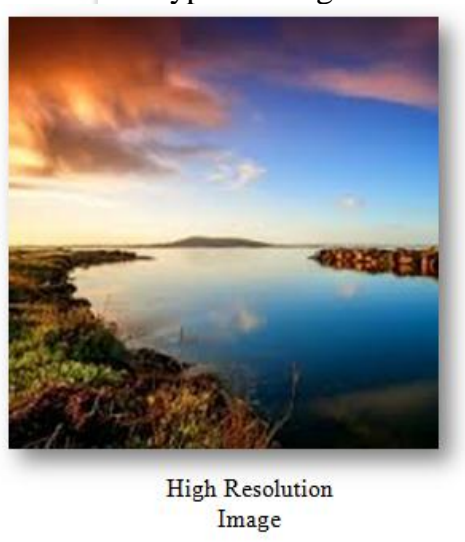

(a)

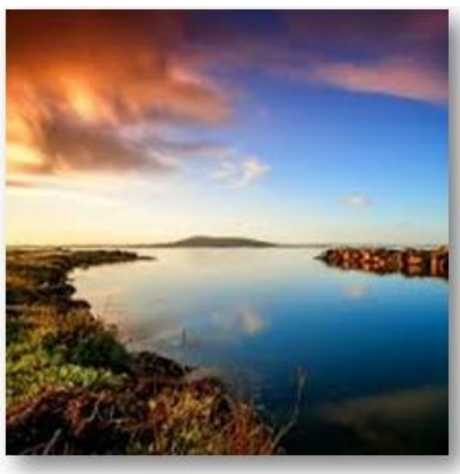

Restored Image (b) 


\section{International Journal of Science and Research (IJSR) \\ ISSN (Online): 2319-7064}

Index Copernicus Value (2013): 6.14 | Impact Factor (2015): 6.391

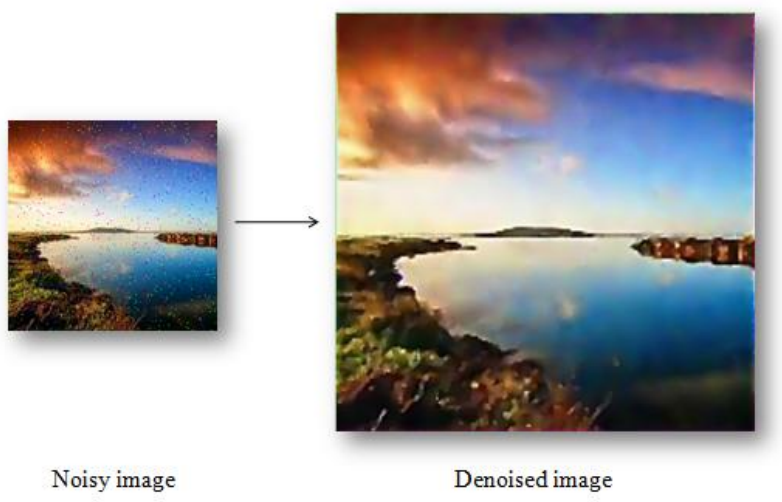

(c)

Figure 3: (a) Interpolation, (b) Restoration with interpolation, (c) De-noising with interpolation

By comparing with the existing similar methodologies, the proposed system performs more efficiently. It results in good accuracy and has high performance.

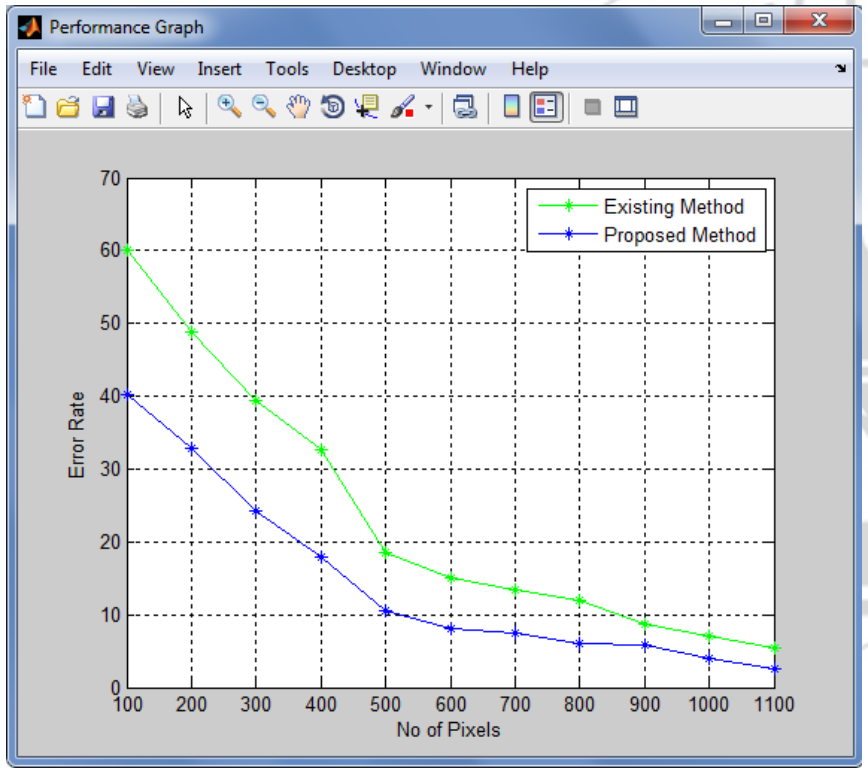

Figure 4: Comparison Graph

A comparison graph is plotted with Error Rate against No of Pixels. The proposed system is compared with an existing system. Finally the proposed method outperforms well. It has a reduced error rate compared with the existing system.

\section{Conclusion}

In this paper a framework for interpolating a torn image is proposed. Initially, the torn image is restored using tear delineation, displacement correction and recovery of the damaged image data. Then the interpolation technique which is based on Low Rank Matrix Completion and Recovery is applied over the restored image to get restored high resolution image. In addition to the restoration of torn image with high quality, the proposed framework also includes a method to restore an image which is affected by noise. That is, if the input image is a noisy one, then the framework uses a denoising algorithm to remove the noise content from the image. The denoising algorithm used here is Block Matching 3- Dimensional (BM3D), which is based on an enhanced sparse representation in transform domain without denoising and with denoising. After denoising the image, convex combination of original noisy image and denoised image is performed. Convex combination is a linear framework which is used to obtain the high quality HR image. This linear combination is performed on orientation and frequency selective bands of the two images. Then the same interpolation technique is applied to get noise-free high resolution image.

\section{References}

[1] Feilong Cao, Miaomiao Cai, and Yuanpeng Tan, "Image Interpolation via Low rank Matrix Completion and Recovery," 2014.

[2] W. T. Freeman, T. R. Jones, and E. C. Pasztor, "Example based super resolution," IEEE Comput. Graph. Appl., vol. 22, no. 2,pp. 56 65, Aug. 2002.

[3] K. Zhang, X. Gao, D. Tao, and X. L. Li, "Single image super resolution with non local means and steering kernel regression," IEEE Trans. Image Process., vol. 21, no. 11, pp. 4544 4556, Jul. 2012.

[4] J. C. Yang, J. Wright, T. Huang, and Y. Ma, "Image superresolution as sparse representation of raw image patches," In Proc. IEEE Comput. Soc. Conf. Comput. Vis. Pattern Recognit., Jun. 2008, pp.1 8.

[5] S. Hou and H. C. Andrews, "Cubic splines for image interpolation and digital filtering," IEEE Trans. Signal Process., vol. 26, no. 6, pp. 508 517, Jan. 1978.

[6] X. Li and M. T. Orchard, "New edge directed interpolation," IEEE Trans. Image Process., vol. 10, no. 10, pp. 1521 1527, Aug. 2001.

[7] E. Cand'es, X. Li, Y. Ma, and J. Wright, "Robust principal omponent analysis?" J. ACM, vol. 58, no. 3, pp. 1 37, May 2011.

[8] Z. Lin, M. Chen, L. Wu, and Y. Ma, "The augmented Lagrange multiplier method for exact recovery of corrupted low rank matrices," preprint arXiv:1009-5055, 2010.

[9] David Corrigan, Anil Kokaram, and Naomi Harte, "Algorithms for the Digital Restoration of Torn Films," IEEE Transactions on Image processing, VOL. 21, NO. 2, Feb 2012.

[10] K. Dabov, A. Foi, V. Katkovnik, and K. Egiazarian. Image denoising by sparse 3-d transform-domain collaborative filtering. IEEE Trans. Image Proc., 2007.

[11] Abhishek Singh1, Fatih Porikli1, and Narendra Ahuja, "Super-Resolving Noisy Images," 2012.

[12] Y. Boykov and M.-P. Jolly, "Interactive graph cuts for optimal boundary region segmentation in n-d images," in Proc. IEEE Int. Conf. Comput. Vis., 2001, vol. 1, pp. 105-112.

[13]R. Coudray and B. Besserer, "Motion based segmentation using MPEG streams and watershedmethod," in Proc. Int. Symp. Vis.Comput., 2005, Lecture Notes in Computer Sciences, pp. 729-736. 


\section{Author Profile}

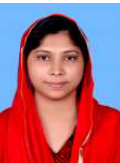

Shadiya $\mathbf{N}$ is pursuing her M.Tech degree in Computer Science and Engineering from KMCT College of Engineering, Calicut University. She obtained her B.Tech Degree in Computer Science and Engineering from College of Engineering, Kallooppara, Cochin University, in 2011.

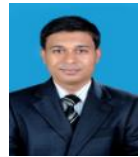

Ameer Ali is Assistant Professor, Department of Computer Science and Engineering, KMCT College of Engineering, Calicut University. He obtained his B.Tech degree in Computer Science \& Engineering from Dr. Pauls Engineering College, Pondicherry in 2011. He completed his M.Tech degree in Computer Science \& Engineering from Reva Institute of Technology and Management, Bangalore in 2014.

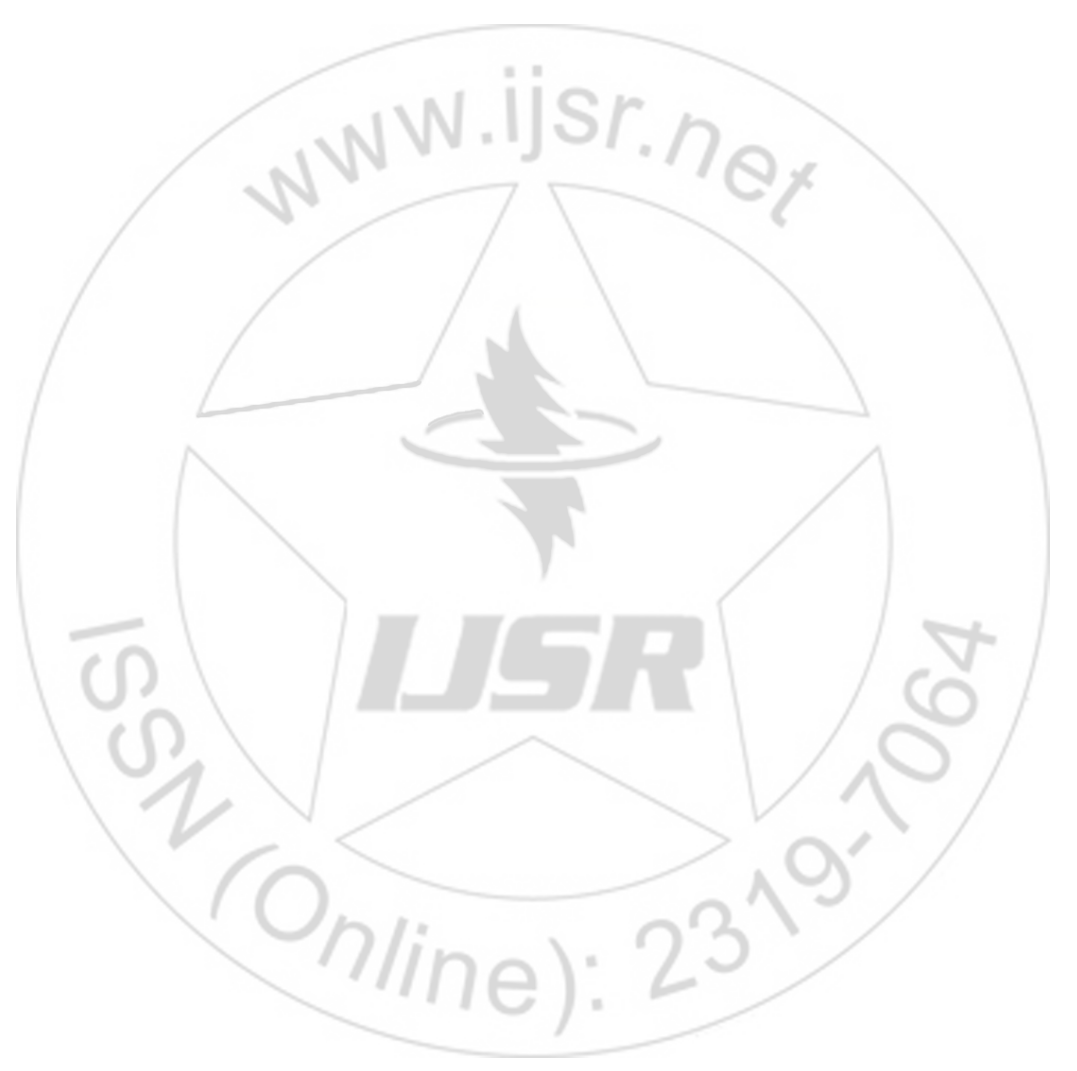

